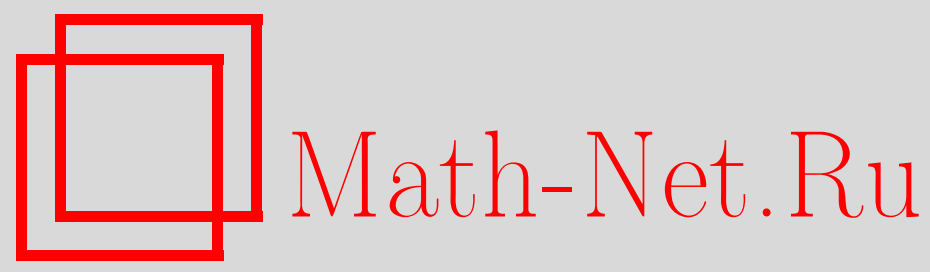

А. А. Туганбаев, Модули над полуцепными кольцами, $M a$ тем. заметки, 1999, том 65, выпуск 6, 880-892

DOI: https://doi.org/10.4213/mzm1124

Использование Общероссийского математического портала Math-Net.Ru подразумевает, что вы прочитали и согласны с пользовательским соглашением http://www . mathnet.ru/rus/agreement

Параметры загрузки:

IP : 18.209.158.208

26 апреля 2023 г., 10:49:54

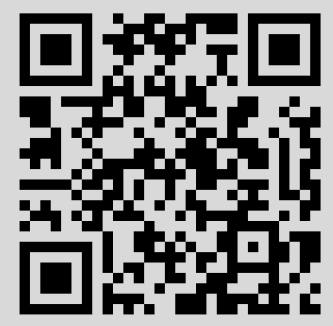




\section{МОДУЛИ НАД ПОЛУЦЕПНЫМИ КОЛЬЦАМИ}

\section{А. А. Туганбаев}

В работе продолжаются исследования полуцепных нётеровых колец. Доказаны общие теоремы о структуре таких колец. В частности, получен ряд резултатов о $\pi$-проективных и $\pi$-инъективных модулях над полуцепными кольцами.

Библиографоя: 14 названий.

Все кольца предполагаются ассоциативньпи и с ненулевой единицей. Слова типа "нётерово кольцо" означают, что соответствующие условия вьполнены справа и слева. Модуль называется иепным, если любые его два подмодуля сравнимы по включению. Прямая сумма цепных модулей назьвается полуиепным модулем. Модуль назьвается наследственным, если все его подмодули проективны. В [1] доказано, что нётерово справа полуцепное полупервичное кольцо является конечным прямьм произведением нётеровых (справа и слева) полуцепных первичных колец. Кроме того, существуют полуцепные справа нётеровы справа полупервичные кольца, не являющиеся ни полуцепньми слева, ни нётеровьми слева кольцами (см., например, [2]). Первым основным результатом данной работы является

Теорема 1. Для кольца А равносильны условия:

(1) A - полуцепное справа нётерово слева полупервичное кольцо;

(2) $A$ - получепное слева нётерово справа полупервичное кольио;

(3) $A$ - конечное прямое произведение полуцепных нётеровых наследственных первичных колеи.

Через $J(M)$ обозначается радикал Джекобсона модуля $M$. Каждьй конечнопорожденньй цепной ненулевой модуль $M$ является локальным модулем (т.е. $M / J(M)$ - простой модуль). Поэтому единица любого полуцепного справа или слева кольца $A$ является суммой локальных ортогональных идемпотентов и, следовательно, $A$ - полусовершенное кольцо. В [3] доказано, что полуцепное кольцо с условием максимальности для правых аннуляторов имеет артиново полуцепное классическое кольцо частных. Вторым основньм результатом данной работы является

Работа выполнена при поддержке Российского фонда фундаментальных исследований, грант № 96-01-00627. 
Теорема 2. Пусть $1=e_{1}+\cdots+e_{n}-$ разложение единицы полуцепного кольиа $A$ в сумму локальных ортогональных идемпотентов $e_{i}$, причем каждое кольцо $e_{i} A e_{i}$ удовлетворяет либо условию максимальности для правых аннуляторов, либо условию максимальности для левых аннуляторов. Тогда А имеет артиново полуиепное классическое кольио частных.

В связи с теоремой 2 отметим, что полуцепные кольца не всегда имеют артиновы классические кольца частных. Пусть $A$ - цепное первичное кольцо с делителями нуля (пример такого кольца см. в [4]). Допустим, что $A$ имеет артиново справа или слева классическое кольцо частных $Q$. Тогда $Q$ является цепньм кольцом. Кроме того, $Q$ изоморфно кольцу матрищ над телом. Поэтому $Q$ - тело и $A$ - область; получено противоречие.

Подмодуль $N$ модуля $M$ назьвается малым (в $M)$, если для любого подмодуля $P$ модуля $M$ из равенства $N+P=M$ следует, что $P=M$. В [5, теорема 4.6] доказано, что каждьй конечнопорожденньй несингулярньй модуль над полуцепным кольцом является проективным модулем. В связи с этим имеет место

Теорема 3. Пусть $M_{A}$ - конечнопорожденный модуль над полуиепным кольиом $A$, причем сингулярный подмодуль $\operatorname{Sing}(M)$ является малым подмодулем в $M$. Тогда $M$ - проективный несингулярный получепной модуль.

Кроме того, у любого несингулярного $A$-модуля все подмодули являются плоскими модулями, причем каждый ингективный несингулярный $A$-модуль является полуцепным модулем.

В связи с теоремой 3 отметим, что циклическая абелева группа $M$ порядка 2 является конечнопорожденным модулем над конечным цепным кольцом вычетов $\mathbb{Z} / 4 \mathbb{Z}$, причем $M$ не является ни проективным, ни несингулярным $\mathbb{Z} / 4 \mathbb{Z}$-модулем.

Кроме теорем 1-3 в работе доказаны другие результаты о полуцепных кольцах. В частности, в п. 3 получен ряд результатов о $\pi$-проективных и $\pi$-инъективных модулях над полуцепными кольцами.

Доказательство теорем разобьем на ряд лемм. Приведем необходимые определения и обозначения. Через $\operatorname{End}(M)$ и $\operatorname{Lat}(M)$ обозначим кольцо эндоморфизмов и решетку подмодулей модуля $M$ соответственно. Через $r_{A}(N)$ обозначим аннулятор в кольце $A$ подмножества $N$ правого модуля $M_{A}$, причем индекс будем опускать, когда ясно, какое кольцо имеется в виду. Элемент $a$ кольца $A$ назьвается регулярным , если $r(a)=l(a)=0$. Через $\operatorname{Sing}(M)$ обозначим сингулярный подмодуль модуля $M_{A}$ над кольцом $A$ (т.е. множество всех элементов $m \in M$ таких, что $r(m)$ - существенный правый идеал кольца $A$ ). Модуль $M_{A}$ называется несингулярным (сингулярным), если $\operatorname{Sing}(M)=0(\operatorname{Sing}(M)=M)$. Модуль $M$ называется дистрибутивным , если Lat $(M)-$ дистрибутивная решетка (т.е. $X \cap(Y+Z)=X \cap Y+X \cap Z$ для любых подмодулей $X, Y, Z$ модуля $M)$. Подмодуль фактормодуля модуля $M$ называется подфактором модуля $M$. Модуль, не содержащий бесконечных прямых сумм ненулевых подмодулей, назьвается конечномерным (в смысле Голди). Модуль назьвается равномерным, если любые два его ненулевых подмодуля имеют ненулевое пересечение. Прямая сумма равномерных (дистрибутивных) модулей назьвается полуравномерны.м (полудистрибутивным) модулем. Модуль $M$ назьвается полунаследственным (риккартовым), если все его конечнопорожденные подмодули (все его циклические подмодули) проективны. Если все конечнопорожденные подмодули модуля $M$ являются нётеровыми, то 
$M$ назьвается локально нётеровым модулем. Модуль $M_{A}$ назьвается точным, если $r(M)=0$ (это равносильно тому, что модуль $A_{A}$ изоморфен подмодулю некоторой прямой степени модуля $M$ ). Модуль $M$ назьвается конечно точным, если существует натуральное число $n$ такое, что прямая сумма $M^{n}$ изоморфных копий модуля $M$ содержит свободный циклический подмодуль. Кольцо $A$ называется совершенным справа, если $A / J(A)$ - артиново кольцо и для любой последовательности $\left\{a_{i}\right\}_{i=1}^{\infty}$ элементов из $J(A)$ существует номер $n$ такой, что $a_{n} \cdot \ldots \cdot a_{1}=0$. Кольцо, не содержащее бесконечных множеств ненулевых ортогональных идемпотентов, называется ортогонально конечным.

\section{1. Доказательство теоремы 1.}

Лемма 1.1. Пусть $M_{A}-$ модуль над кольцом А. Тогда

(1) если $M$ - конечномерный модуль, $M=\bigoplus_{i=1}^{n} M_{i}$ и $M_{i}$ - риккартовы (полунаследственные) модули, то $M$ - риккартов (полунаследственны й) мо$\partial у л ь$

(2) если $M-$ - риккартов модуль, то $M$ - несингулярный модуль.

ДоказАтельство. (1) Пусть $h_{i}: M \rightarrow M_{i}$ - естественные проекции, все $M_{i}$ - риккартовы (полунаследственные) модули и $N$ - ненулевой циклический (конечнопорожденньй) подмодуль модуля $M$. По предположению все $h_{i}(N)$ - проективные модули, откуда $N=N_{i} \oplus N \cap\left(\bigoplus_{j \neq i} M_{i}\right)$, где $N_{i} \cong h_{i}(N)(i=1, \ldots, n)$. Осталось провести индукцию по $n$, и утверждение будет доказано.

(2) Пусть $m$ - элемент модуля $M$ такой, что $r(m)$ - существенньй правый идеал кольца $A$. Так как $m A$ - проективньй модуль, изоморфньй модулю $(A / r(a))_{A}$, то $r(m)-$ существенное прямое слагаемое модуля $A_{A}$. Тогда $r(a)=A, m=0$.

Лемма 1.2. (1) Если е - идемпотент несингулярного справа кольца $A$ такой, что еА - равномерный правый $A$-модуль, то $r(a е)=(1-e) A$ для любого ненулевого ае $\in$ Ае и, следовательно, ае $A_{A}-$ проективный модуль.

(2) Если $A$ - риккартово справа конечномерное справа полупервичное кольио, то $A$ - полуравномерное справа кольцо.

(3) Если $A$ - полупервичное кольцо с условием максимальности для правых аннуляторов, то $A$ - несингулярное слева кольцо.

(4) Если $A$ - кольцо с условием максимальности для правых аннуляторов, то $\operatorname{Sing}\left(A_{A}\right)$ - нильпотентный идеал.

ДоказАтельство. (1) Так как $(1-e) A \subseteq r(a e)$, то $r(a e)=e B \oplus(1-e) A$, где $e B$ - подмодуль модуля $e A$. Допустим, что $(1-e) A \neq r(a e)$. Тогда $e B \neq 0$. Так как $e B$ - ненулевой подмодуль равномерного модуля $e A$, то $e A$ - сушественное расширение модуля $e B$. Поэтому $r(a e)=e B \oplus(1-e) A$ - сушественньй правый идеал кольца $A$. Так как $A$ несингулярно справа, то $a e=0$; получено противоречие.

(2) Риккартово справа кольцо $A$ несингулярно справа. Конечномерное справа кольцо $A$ имеет существенньй правый идеал $M$, являющийся конечной прямой суммой равномерных правых идеалов $M_{1}, \ldots, M_{n}$. Так как $A$ - несингулярное справа конечномерное справа полупервичное кольцо, то $A$ - правьй порядок в полупростом артиновом кольце $[6,3.32,3.35]$. Поэтому существенньй правьй идеал $M$ кольца содержит некоторьй регулярный элемент $a$. Так как $A$ - риккартово справа конечномерное справа кольцо и $a A \subseteq M$, то проективньй модуль $a A$ изоморфен прямой сумме некоторых подмодулей из $M_{i}$, причем $a A \cong A_{A}$. Поэтому $A$ - полуравномерное справа кольцо. 
(3) Пусть $H \equiv \operatorname{Sing}\left({ }_{A} A\right)$. Так как $A$-кольцо с условием максимальности для правых аннуляторов, то $A$ - кольцо с условием минимальности для левых аннуляторов. Поэтому существуют элементы $h_{1}, \ldots, h_{m} \in H$ такие, что $l(H)=\bigcap_{i=1}^{n} l\left(h_{i}\right)$. Тогда $l(H)-$ существенный левый идеал. Так как $(H \cap l(H))^{2}=0$ и $A$ - полупервичное кольцо, то $H \cap l(H)=0$. Поскольку $l(H)$ - существенньй левьй идеал, то $H=0$.

(4) доказано в $[6,3.31]$.

Лемма 1.3. Пусть $A$ - полусовершенное кольцо, у которого все 2-порожденные правые идеалы проективны. Тогда

(1) если $A$ - полуравномерное справа кольцо, то $A$ - получепное справа кольцо;

(2) если $A$ - конечномерное справа полупервичное кольцо, то $A$ - получепное справа кольчо.

ДокАЗАТЕЛьСтво. (1) Пусть $M$ - равномерное прямое слагаемое модуля $A_{A}$. Достаточно доказать, что $M_{A}$ - цепной модуль. Допустим противное. Существуют несравнимые по включению циклические подмодули $F$ и $G$ модуля $M$. Тогда $F+G-$ нелокальный 2 -порожденньй модуль. Так как $F+G$ - неразложимьй конечнопорожденньй проективньй модуль над полусовершенным кольцом $A$, то $F+G$ - локальный модуль (см. [7, 22.23]). Получено противоречие.

(2) следует из (1) и леммы $1.2(2)$.

Лемма 1.4. (1) Пусть все матричные кольиа $A_{n}$ над кольчом $А$ ортогонально конечны. Тогда $A$ полунаследственно справа $\Longleftrightarrow A$ полунаследственно слева.

(2) Пусть $A$ - конечномерное справа или слева кольцо. Тогда $A$ полунаследственно справа $\Longleftrightarrow A$ полунаследственно слева.

ДокАЗАТЕЛьСтво. (1) доказано в [8, теорема 3].

(2) Согласно (1) достаточно доказать, что все матричные кольца $A_{n}$ ортогонально конечны. Так как $A$ конечномерно справа или слева, то либо все конечнопорожденные проективные правые $A$-модули конечномерны, либо все конечнопорожденные проективные левые $A$-модули конечномерны. Поэтому все кольца $A_{n}$ конечномерны справа или слева. В частности, все кольца $A_{n}$ ортогонально конечны.

Лемма 1.5. Пусть $A-$ конечномерное справа наследственное справа кольцо. Тогда

(1) $A$ - нётерово справа кольцо;

(2) если $B$ - конечнопорожденный существенный левый идеал кольца $A$, то

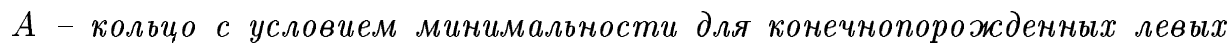
идеалов, содержаших $B$;

(3) если $A$ - конечномерное слева кольчо и $B$ - существенный левый идеал кольиа $A$, то $A$ - кольцо с условием минимальности для конечнопорожсденных левых идеалов, содержащих $B$;

(4) если е - ненулевой идемпотент кольиа $A$ такой, что $A$ - иепной левый $A$-модуль и $b$ - ненулевой элемент из $A e$, то $A e / A b$ - артинов левый A-модуль.

ДокАЗАТЕЛЬСтво. (1) доказано в [6, следствие 5.20].

(2) Согласно (1) $A$ - нётерово справа кольцо. Для нётерова справа наследственного справа кольца $A$ утверждение доказано в [9, теорема 2.2]. 
(3) следует из (2) и того, что каждьй конечномерньй модуль является существенным расширением конечнопорожденного модуля.

(4) Пусть $B \equiv A b \oplus A(1-e)$. Так как цепной модуль $A e-$ сушественное расширение модуля ${ }_{A} A b$, то $B$ - конечнопорожденный существенньй левьй идеал. Согласно (2) $A-$ кольцо с условием минимальности для конечнопорожденных левых идеалов, содержащих $B$. Кроме того, модуль $A A / B$ изоморфен цепному модулю $A e / A b$. Поэтому $A e / A b-$ артинов модуль.

ПРЕДЛОЖЕНИЕ 1.6. Для полуцепного справа кольца А равносильны условия:

(1) $A$ - несингулярное справа кольцо;

(2) $A$ - полунаследственное справа кольио;

(3) $A$ - полунаследственное слева кольцо.

ДокАЗАТЕЛЬСтво. $(2) \Longleftrightarrow(3)$ следует из леммы $1.4(2) .(2) \Longrightarrow(1)$ следует из леммы $1.1(2)$.

$(1) \Longrightarrow(2)$. Пусть $1=e_{1}+\cdots+e_{n}$, где $e_{i}$ - ненулевые ортогональные идемпотенты такие, что все $e_{i} A$ - цепные правые $A$-модули. Рассмотрим некоторое $e_{i} \equiv e$. По лемме 1.1 (1) достаточно доказать, что $e A$ - полунаследственньй $A$-модуль. Пусть $B-$ конечнопорожденньй подмодуль модуля $e A$. Так как $e A-$ цепной модуль, то $B=e b A$ для некоторого элемента $e b \in e A$. Тогда $\sum_{j=1}^{n} e b e_{j} A=e b A$ - подмодуль цепного модуля $e A$. Поэтому существует индекс $j$ такой, что

$$
e^{b} e_{j} A=\sum_{k=1}^{n} e b e_{k} A=e b A .
$$

По лемме $1.2(1)$ ebe ${ }_{j} A=B$ - проективный модуль.

Лемма 1.7. Пусть $A-$ полуцепное слева кольио. Тогда

(1) $A / \bigcap_{n=1}\left(J(A)^{n}\right)$ - нётерово слева кольцо, причем $A / J(A)^{n}$ - артиново слева кольцо для любого натурального числа $n$;

(2) для любых примитивных ненулевых идемпотентов е $u f$ кольиа $A$ верно, что ${ }_{A}$ Aе и ${ }_{A} A f$ - цепные левые $A$-модули, еАе - чепное слева кольио и еAf - иепной левы й еАе-модуль;

(3) если $A$ - несингулярное слева кольцо, то $A$ - полунаследственное (справа и слева) кольио;

(4) если $A$ - полупервичное кольцо, то $A$ - конечное прямое произведение полуцепных слева первичных колеи;

(5) если $A$ - конечномерное справа наследственное справа кольцо и $B$ - существенный левый идеал кольиа $A$, то ${ }_{A} A / B$ - артинов модуль.

ДоказАтельство. (1) Пусть $M \equiv J(A)$. Так как $A / M^{n}$ - полуцепное слева полупримарное кольцо, то непосредственно проверяется, что $A / M^{n}$ - артиново слева кольцо. Докажем, что $A / \bigcap_{n=1}\left(J(A)^{n}\right)$ - нётерово слева кольцо. Без ограничения общности можно считать, что $\bigcap_{n=1} M^{n}=0$. Пусть $1=e_{1}+\cdots+e_{n}$, где $e_{i}$ - ненулевые ортогональные идемпотенты такие, что все $e_{i} A$ - цепные правые $A$-модули. Рассмотрим некоторый идемпотент $e_{i} \equiv e$. Достаточно доказать, что $e A$ - нётеров $A$-модуль. Пусть $B$ - ненулевой подмодуль в $e A$. Надо доказать, что $B$ - конечнопорожденньй модуль. Так как $\bigcap_{n=1} M^{n}=0$, то $B \nsubseteq M^{n}$ для некоторого $n$. Поскольку $e A-$ цепной модуль, 
то $B$ строго содержит $M^{n} \cap e A=e M^{n}$. Поэтому $M^{n} \subseteq B \oplus(1-e) A$. Факторкольцо $A / M^{n}$ артиново справа и, в частности, нётерово справа. Поэтому $B / e M^{n} \oplus(1-e) A /$ $(1-e) M^{n}$ - конечнопорожденный правый $A$-модуль, откуда $B / e M^{n}$ - конечнопорожденньй правый $A$-модуль. Кроме того, $e M^{n}$ - малый подмодуль цепного модуля $B_{A}$. Поэтому $B_{A}$ - конечнопорожденньй $A$-модуль.

(2) Так как любое разложение полусовершенного кольца в прямую сумму неразложимых ненулевых левых идеалов является единственным с точностью до изоморфизма и $A$ - полуцепное слева кольцо, то ${ }_{A} A e$ и ${ }_{A} A f$ - цепные модули. Тогда непосредственно проверяется, что $e A f$ - цепной левьй $e A e$-модуль. В частности, $e A e-$ цепное слева кольцо.

(3) следует из симметричного аналога предложения 1.6.

(4) Единица полусовершенного кольца $A$ является суммой локальных ортогональных идемпотентов $e_{1}, \ldots, e_{m}$. Так как $A$ - конечномерное слева полупервичное кольцо, то существуют несравнимые по включению первичные идеалы $P_{1}, \ldots, P_{n}$ такие, что $P_{1} \cap \cdots \cap P_{n}=0$. Теперь достаточно доказать, что $P_{i}+P_{j}=A$ при $i \neq j$. А именно, для каждого $e_{k}$ и любых несовпадающих $i, j$ доказать включение $e_{k} \in A \backslash P_{i}+P_{j}$. Положим $e \equiv e_{k}$. Согласно (2) eAe- цепное слева кольцо. Поэтому идеалы $e P_{i} e$ и $e P_{j} e$ кольца $е A$ с сравнимы по включению. Например, пусть $e P_{i} e \subseteq e P_{j} e \subseteq P_{j}$. Так как $P_{j}-$ первичный идеал и $P_{i} \nsubseteq P_{j}$, то $e \in P_{j} \subseteq P_{i}+P_{j}$.

(5) Пусть ${ }_{A} A=\sum_{i=1}^{n} A_{i}$, где все $A_{i}$ - цепные левые $A$-модули. Так как $B$ - существенный левый идеал, то существуют ненулевые элементы $b_{i} \in A_{i} \cap B$. По лемме $1.5(4)$ все левые $A$-модули $A_{i} / A b_{i}$ являются артиновыми. Пусть $C \equiv \sum_{i=1}^{n} A b_{i}$. Так как ${ }_{A} A / C \cong \bigoplus_{i=1}^{n} A_{i} / A b_{i}$, то модуль ${ }_{A} A / C$ артинов. Поэтому ${ }_{A} A / B$ - артинов модуль.

ПРЕДЛОЖЕНИЕ 1.8. (1) Пусть $A$ - полуцепное справа кольцо. Тогда $A$ - нёте-

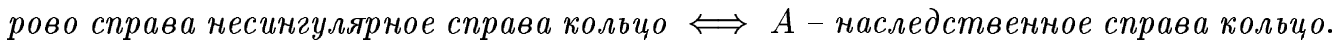

(2) Пусть $A$ - получепное кольио. Тогда $A$-несингулярное справа кольцо $\Longleftrightarrow$ $A-$ несингулярное слева кольио $\Longleftrightarrow A-$ полунаследственное кольио.

(3) Если $A$ - нётерово справа несингуляное справа полуцепное кольцо, то $A-$ наследственное справа кольцо и для каждого существенного левого идеала $B$ кольиа $A$ модуль ${ }_{A} A / B$ является артиновым.

ДокАЗАТЕЛЬСТВо. (1) следует из предложения 1.6 и леммы 1.5 (1).

(2) следует из предложения 1.6.

(3) Согласно (1) $A$-наследственное справа кольцо. По лемме $1.7(5){ }_{A} A / B$-артинов модуль.

ДокАЗАТЕЛЬСТво ТЕОРЕмЫ 1. Так как условие (3) симметрично, то достаточно доказать $(2) \Longleftrightarrow(3)$. Импликация $(3) \Longrightarrow(2)$ очевидна.

$(2) \Longrightarrow(3)$. По лемме $1.7(4)$ можно без ограничения общности считать, что $A-$ первичное кольцо. По лемме $1.2(3) A$ несингулярно слева. По лемме $1.7(3) A$ - полунаследственное кольцо. Так как $A$ - нётерово справа полунаследственное справа кольцо, то $A$ наследственно справа. Наследственное справа полусовершенное первичное кольцо $A$ является по лемме $1.3(2)$ полуцепным справа. Пусть $M \equiv J(A), B \equiv \bigcap_{n=1} M^{n}$. Можно считать, что $M \neq 0$. Так как $A$ - нётерово справа кольцо, то по лемме Накаямы $M^{n} \neq M^{n+1}$ для любого $n$. Поэтому $A / B$ не является артиновым слева кольцом. Допустим, что $B \neq 0$. Так как $A$ - первичное кольцо, то его ненулевой идеал $B$ является существенным левьг идеалом. По лемме $1.7(5){ }_{A} A / B$ - артинов модуль. Тогда 
$A / B$ - артиново слева кольцо; получено противоречие. Поэтому $B=0$. Так как по лемме $1.7(1) A / B$ - нётерово слева кольцо, то $A$ - нётерово слева кольцо. Нётерово слева полунаследственное слева кольцо $A$ является наследственным слева кольцом.

\section{2. Доказательство теоремы 2.}

Лемма 2.1 [10, лемма 3]. Для модуля $M_{A}$ равносильны условия:

(1) $М-$ - истрибутивный модуль;

(2) для любых әлементов $m$ и и из $M$ существуют әлементы $a, b, c, d$ кольца $A$ maкuе, что $1=a+b, m a=n c, n b=m d$.

Лемма 2.2. Пусть $Q$ - правое кольцо частных кольиа А относительно правого мнохсества Оре $T$ в А. Тогда

(1) если $M$ - правый идеал в $A$ такой, что $M_{A}-$ дистрибутивный (чепной) модуль, то $M Q_{Q}-$ дистрибутивный (иепной) модуль;

(2) если единица кольиа $A$ является суммой ненулевых идемпотентов $e_{1}, \ldots, e_{n}$

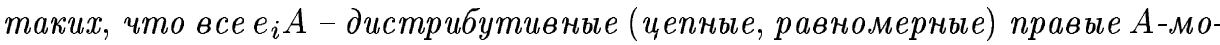
дули, то все $e_{i} Q$ - дистрибутивные (цепные, равномерные) правые модули над кольиом $Q$

(3) если $A$ - полудистрибутивное справа (полуцепное справа, полуравномерное справа) кольцо, то $Q$ - полудистрибутивное справа (получепное справа, полуравномерное справа) кольцо.

ДоказАтЕльство. (1) Пусть $q_{1}$ и $q_{2}$ - любые ненулевые элементы из $M Q$. Существуют ненулевые элементы $m_{1}, m_{2} \in M$ и $t \in T$ такие, что $q_{i}=m_{i} t^{-1}(i=1,2)$.

Допустим, что $M_{A}$ дистрибутивен. По лемме 2.1 существуют $a_{1}, a_{2}, b_{1}, b_{2} \in A$ такие, что $1=a_{1}+a_{2}, m_{1} a_{1}=m_{2} b_{2}$ и $m_{2} a_{2}=m_{1} b_{1}$. Тогда $1=t a_{1} t^{-1}+t a_{2} t^{-1}, q_{1}\left(t a_{1} t^{-1}\right)=$ $m_{1} a_{1} t^{-1}=m_{2} b_{2} t^{-1}=q_{2}\left(t b_{2} t^{-1}\right)$ и $q_{2}\left(t a_{2} t^{-1}\right)=m_{2} a_{2} t^{-1}=m_{1} b_{1} t^{-1}=q_{1}\left(t b_{1} t^{-1}\right)$. По лемме $2.1 M Q_{Q}$ дистрибутивен.

Допустим, что $M_{A}$ равномерен. Тогда существуют $a_{1}, a_{2} \in A$ такие, что $0 \neq m_{1} a_{1}=$ $m_{2} a_{2}$. Поэтому $0 \neq m_{1} a_{1} t^{-1}=m_{2} a_{2} t^{-1}=q_{1}\left(t a_{1} t^{-1}\right)=q_{2}\left(t b_{2} t^{-1}\right)$. Тогда $M Q_{Q}$ равномерен.

Допустим, что $M_{A}$ - цепной модуль и $q_{2} \in M Q \backslash q_{1} Q$. Так как $q_{2} \in M Q \backslash q_{1} Q$, то $m_{2} \in M \backslash m_{1} A$. Поскольку $M_{A}$ - цепной модуль, существует $a \in A$ такое, что $m_{1}=m_{2} a$. Тогда $q_{2}\left(t a t^{-1}\right)=m_{2} a t^{-1}=m_{1} t^{-1}=q_{1}$. Поэтому $M Q_{Q}$ - цепной модуль.

(2) и (3) следуют из (1).

ЛЕмма 2.3. Пусть единица кольца $A$ является суммой ненулевых идемпотентов $e_{1}, \ldots, e_{n}$ таких, что все $A e_{i}$ - равномерные левые идеаль кольиа $A$ и все $e_{i} A$ - равномерные правые идеаль кольиа $A ; L_{i j}$ - множсество всех әлементов $e_{i} a e_{j}$ из $e_{i} A e_{j}$ таких, что $\left(e_{i} a e_{j}\right)\left(e_{j} b\right)=0$ для некоторого $e_{k} u$ некоторого ненулевого әлемента $e_{j} b$ в $e_{j} A ; R_{i j}$ - мноэсество всех элементов $e_{i} a e_{j}$ из $e_{i} A e_{j}$ таких, что $\left(\right.$ Ace $\left._{i}\right)\left(e_{i} a e_{j}\right)=0$ для некоторого ненулевого әлемента се $e_{i}$ в $A e_{i} ; u T_{i j} \equiv e_{i} A e_{j} \backslash\left(L_{i j} \cup R_{i j}\right)$. Тогда

(1) $L_{i j}$ и $R_{i j}$ - левые $e_{i} A e_{i}$-модули и правые $e_{j} A e_{j}$-модули;

(2) ecлu $\left(e_{i} v e_{j}\right)\left(e_{j} w e_{i}\right) \in T_{i i}$, mo $_{i} v e_{j} \in T_{i j} u e_{j} w e_{i} \in T_{j i}$. 
ДокаЗАтельство. (1) Пусть $0 \neq e_{i} x e_{i} \in e_{i} A e_{i}, 0 \in e_{j} y e_{j} \in e_{j} A e_{j}, e_{i} a e_{j} \in L_{i j}$ и $\left(e_{i} a e_{j}\right)\left(e_{j} b\right)=0$ для некоторого ненулевого $e_{j} b$ в $e_{j} A$. Тогда $\left(e_{i} x e_{i}\right)\left(e_{i} a e_{j}\right)\left(e_{j} b\right)=0$, откуда $\left(e_{i} A e_{i}\right) L_{i j} \subseteq L_{i j}$. Так как $e_{j} A$ - равномерньй правый идеал в $A$, то $e_{j} b A \cap$ $e_{j} y e_{j} A \neq 0$. Таким образом, сушествует ненулевой элемент $e_{j} c \in e_{j} A$ такой, что $0 \neq$ $\left(e_{j} y e_{j}\right)\left(e_{j} c\right) \in e_{j} b A$. Тогда $\left(e_{i} a e_{j}\right)\left(e_{j} y e_{j}\right)\left(e_{j} c\right)=0$, откуда $\left(e_{i} a e_{j}\right)\left(e_{j} y e_{j}\right) \in L_{i j}$. Поэтому $L_{i j}\left(e_{j} A e_{j}\right) \subseteq L_{i j}$. Аналогично, $e_{i} A e_{j} L_{i j}\left(e_{j} A e_{j}\right) \subseteq L_{i j}$.

(2) Докажем, что $e_{i} v e_{j} \in T_{i j}$. Предположим противное. Тогда либо $e_{i} v e_{j} \in L_{i j}$, либо $e_{i} v e_{j} \in R_{i j}$. Допустим, что $e_{i} v e_{j} \in L_{i j}$. Тогда $\left(e_{i} v e_{j}\right)\left(e_{j} b\right)=0$ для некоторого ненулевого $e_{j} b$ из $e_{j} A$. Так как $\left(e_{i} v e_{j}\right)\left(e_{j} w e_{i}\right) \in T_{i i}$, то $e_{j} w e_{i} \neq 0$. Поскольку $e_{j} A-$ равномерньй правый идеал в $A$, то $e_{j} b A \cap e_{j} w e_{i} A \neq 0$. Поэтому существует ненулевой элемент $e_{i} c \in e_{i} A$ такой, что $0 \neq\left(e_{j} w e_{i}\right)\left(e_{i} c\right) \in e_{j} b A$. Тогда $\left(e_{i} v e_{j}\right)\left(e_{j} w e_{i}\right)\left(e_{i} c\right)=0$, откуда $\left(e_{i} v e_{j}\right)\left(e_{j} w e_{i}\right) \in L_{i i} ;$ получено противоречие с включением $\left(e_{i} v e_{j}\right)\left(e_{j} w e_{i}\right) \in$ $T_{i i}$. Аналогично, невозможно включение $e_{i} v e_{j} \in R_{i j}$. Поэтому $e_{i} v e_{j} \in T_{i j}$. Включение $e_{j} w e_{i} \in T_{j i}$ доказьвается аналогично.

Лемма 2.4. (1) Если $M_{A}$ - дистрибутивный правый модуль над совершенным справа (слева) кольчом $A$, то $M$ - нётеров (артинов) модуль.

(2) Если A - полудистрибутивное справа совершенное справа или слева кольио, то А - артиново справа кольио.

(3) Пусть единица кольиа $A$ является суммой ненулевых ортогональных идемпотентов $e_{1}, \ldots, e_{n}$ таких, что все $e_{i} A e_{i}-$ совершенные справа или слева кольиа, причем все $e_{j} A e_{i}$ - дистрибутивные правые $e_{i} A e_{i}$-модули. Тогда $A$ - артиново справа кольцо.

ДоказАТЕЛЬСТво. (1) доказано в [11].

(2) Так как конечная прямая сумма нётеровых (артиновых) модулей - нётеров (артинов) модуль, то согласно (1) $A$ - либо нётерово справа совершенное справа кольцо, либо артиново справа кольцо. Заметим, что любое нётерово справа совершенное справа кольцо является артиновьм справа.

(3) Обозначим через $R$ унитарное подкольцо $e_{1} A e_{1} \times \cdots \times e_{n} A e_{n}$ кольца $A$. Согласно (2) все кольца $e_{i} A e_{i}$ артиновы справа. Поэтому $R$ артиново справа. В силу (1) все $e_{j} A e_{i}$ - артиновы правые $e_{i} A e_{i}$-модули. Поэтому все $e_{j} A e_{i}$ - артиновы правые $R$-модули. Следовательно, $A$ - артинов правьй модуль над его унитарньм подкольцом $R$. Значит, $A$ - артиново справа кольцо.

Лемма 2.5. Пусть $1=e_{1}+\cdots+e_{n}-$ разложсение единицы полуцепного кольца $А$ в сумму локальных ортогональных идемпотентов $e_{i}$. Сопоставим каждому әлементу $a \in A$ матрииу $M(a)=\left(e_{i} a e_{j}\right)$. Тогда

(1) $M(a b)=M(a) \cdot M(b)$ для всех $a, b \in A$;

(2) для каждого әлемента $a \in A$ существуют обратимые әлементы $u, v \in A$ такие, что все строки и столбиы матрицы $M($ иаv) содержат не более одного ненулевого әлемента;

(3) для каждого әлемента $a \in A$ существуют обратимые әлементы $u, v \in A$ и натуральное число $m$ такие, что $(\text { иаv })^{m}=e_{1}($ uav $) e_{1}+\cdots+e_{m}($ uav $) e_{m}$ (т.е. $M\left((\text { uаv })^{m}\right)$ - диагональная матрича).

ДокАЗАТЕЛЬСТво. (1) проверяется непосредственно.

(2) является частньм случаем леммы 3 из [12]. 
(3) В силу (2) существуют обратимые элементы $u, v \in A$ такие, что все строки и столбцы матрицы $M(u a v)$ содержат не более одного ненулевого элемента. Определим перестановку $\pi$ на множестве $N \equiv\{1, \ldots, n\}$. Если для $i \in N$ существует $j \in N$ такое, что $e_{i}(u a v) e_{j} \neq 0$, то полагаем $\pi(i)=j$. Если $i \in N$, причем $e_{i}(u a v) e_{j}=0$ для всех $j \in N$, то полагаем $\pi(i)=2^{i+n}$. Существует $m$ такое, что $\pi^{m}$ - тождественная перестановка. Тогда $M\left((u a v)^{m}\right)$ - диагональная матрища. Поэтому $(u a v)^{m}=e_{1}(u a v) e_{1}+\cdots$ $+e_{m}(u a v) e_{m}$

ЛЕмма 2.6. Пусть $А$-иепное кольцо с условием максимальности для правых аннуляторов. Тогда

(1) $A$ - кольцо с условием максимальности для левых аннуляторов;

(2) А имеет иепное артиново классическое кольио частных $Q$.

ДоКАЗАТЕЛЬСТвО. Пусть $N$ и $N^{*}$ - множества всех левых делителей нуля и всех правых делителей нуля в $A$ соответственно. Так как $A$-равномерное кольцо, то $N$ и $N^{*}$ совпадают с правьп сингулярным идеалом $\operatorname{Sing}\left(A_{A}\right)$ и левым сингулярным идеалом $\operatorname{Sing}\left({ }_{A} A\right)$ кольца $A$ соответственно, откуда $N$ и $N^{*}$ - вполне первичные идеалы в $A$. Так как $A$ - кольцо с условием максимальности для правых аннуляторов, то по лемме $1.2(4)$ правый сингулярньй идеал $N$ нильпотентен. Цепная область $A / N$ является порядком в теле $Q$. Без ограничения общности можно считать, что $N$ - ненулевой идеал.

(1) Допустим противное. Тогда $A$ имеет бесконечную убывающую цеп $B_{1} \supset B_{2} \supset \cdots$ правых идеалов $B_{i}$ такую, что $l\left(B_{1}\right) \subset l\left(B_{2}\right) \subset \cdots-$ бесконечная возрастающая цепь левых аннуляторов. Так как $N$ содержит все левые делители нуля в $A$, то $l\left(B_{i}\right) \subseteq N$ для всех $i$. Поскольку $N$ - ненулевой нильпотентньй идеал и $A$ - цепное слева кольцо, существует натуральное число $n$ такое, что $N^{n+1} \subset l\left(B_{i}\right) \subset l\left(B_{i+1}\right) \subset N^{n}$, где все включения являются строгими. Пусть $a \in l\left(B_{i+1}\right) \backslash l\left(B_{i}\right)$. Тогда $a B_{i+1}=0$ и $a B_{i} \neq 0$. Так как $A$ - цепное слева кольцо и $l\left(B_{i}\right) \subset A a$, существует левый идеал $D$ в $A$ такой, что $l\left(B_{i}\right)=D a$. Если $D \subseteq N$, то $l\left(B_{i}\right) \subseteq D N^{n} \subseteq N^{n+1} ;$ получено противоречие. Поэтому $D$ строго содержит $N$. Пусть $d \in D \backslash N$. Тогда $r(d)=0$ и $d a B_{i}=0$. Поэтому $a B_{i}=0$; получено противоречие.

(2) Так как согласно (1) $A$ - кольцо с условием максимальности для левых аннуляторов, то по лемме $1.2(4)$ левый сингулярньй идеал $N^{*}$ нильпотентен. Поскольку $N$ и $N^{*}$ - вполне первичные нильпотентные идеалы, то $N=N^{*}$. Поэтому $A \backslash N$ совпадает с множеством $T$ всех регулярных элементов из $A$. Кроме того, $A$ - цепное кольцо. Тогда $A$ имеет классическое кольцо частных $Q$, являющееся по лемме $2.2(1)$ цепным кольцом. Пусть $t \in A \backslash N$. Так как $A$ - цепное кольцо и $N$-вполне первичньй идеал, то $N=t N=N t$, откуда $t^{-1} N=N=N t^{-1}$. Поэтому $N=N Q=Q N$ - нильпотентньй идеал в кольце $Q$ и все элементы из $Q \backslash N$ обратимы в $Q$. Следовательно, $Q$ - цепное полупримарное кольцо. Тогда $Q$ - артиново кольцо.

ЛЕмма 2.7. Пусть $1=e_{1}+\cdots+e_{n}-$ разложение единицы полуцепного кольиа $A$ в сумму локальных ортогональных идемпотентов $e_{i}, T_{i}$ - множество всех регулярных әлементов в кольце $e_{i} A e_{i}, T$-множество всех әлементов $t$ в $A$ вида $t=t_{1}+\cdots+t_{n}$, где $t_{i} \in T_{i}$. Тогда

(1) $T$ - множсество Оре в $A$, кольцо частных $Q$ кольца $A$ относительно $T$ является полуиепным кольиом, все $e_{i} Q$ - иепные $Q$-модули, все $e_{i}$ - локальные идемпотенты в $Q$, все $e_{i} A e_{i}$ и $e_{i} Q e_{i}-$ чепные кольиа, $e_{i} T e_{i}=T_{i}$ 


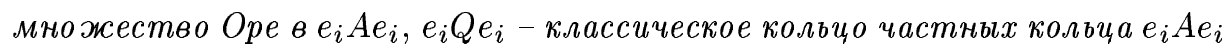
и $e_{i} Q e_{i}-$ кольцо частных кольца $e_{i} A e_{i}$ относительно $e_{i} T e_{i}$ для любого $i$;

(2) полуцепное кольцо частных $Q$ кольца $A$ относительно мнохества $T$ является классическим кольиом частных $Q_{\mathrm{cl}}(A)$ кольиа $A$;

(3) если каждое кольцо $e_{i} A e_{i}$ является либо кольцом с условием максимальности для правых аннуляторов, либо кольцом с условием максимальности для левых аннуляторов, то А имеет артиново получепное классическое кольцо частных $Q_{\mathrm{cl}}(A)$, совпадающее с кольиом частных $Q$ относительно множсества $T$.

ДокАЗАТЕЛЬСтво. (1) По лемме $2.2(3)$ достаточно доказать, что $T$ - множество Ope. Пусть $L_{i j}-$ множество всех элементов $e_{i} a e_{j}$ из $e_{i} A e_{j}$ таких, что $\left(e_{i} a e_{j}\right)\left(e_{j} b e_{k}\right)=0$ для некоторого $e_{k}$ и некоторого ненулевого $e_{j} b e_{k}$ из $e_{j} A e_{k} ; R_{i j}$ - множество всех элементов $e_{i} a e_{j}$ из $e_{i} A e_{j}$ таких, что $\left(e_{m} c e_{i}\right)\left(e_{i} a e_{j}\right)=0$ для некоторого $e_{m}$ и некоторого ненулевого элемента $e_{m} c e_{i}$ из $e_{m} A e_{i} ; T_{i j} \equiv e_{i} A e_{j} \backslash\left(L_{i j} \cup R_{i j}\right)$.

Пусть $a \in A, t \in T$. Докажем, что для любых $i$ и $j$ существует элемент $e_{j} u(i, j) e_{j} \in T_{j j}$ такой, что $\left(e_{i} a e_{j}\right)\left(e_{j} u(i, j) e_{j}\right) \in e_{i} t e_{i} A$. Так как $e_{i} A$-цепной правьй $A$-модуль, то либо $e_{i} a e_{j} \in e_{i} t e_{i} A$, либо $e_{i} t e_{i} \in e_{i} a e_{j} A$. Если $e_{i} a e_{j} \in e_{i} t e_{i} A$, то $\left(e_{i} a e_{j}\right) e_{j} \in e_{i} t e_{i} A, e_{j} \in$ $T_{j j}$, и можно положить $e_{j} u(i, j) e_{j} \equiv e_{j}$. Допустим, что $e_{i} t e_{i}=\left(e_{i} a e_{j}\right) b \in e_{i} a e_{j} A$, где $b \in A$. Тогда $e_{i} t e_{i}=\left(e_{i} a e_{j}\right)\left(e_{j} b e_{i}\right)$. По лемме $2.3(2) e_{i} a e_{j} \in T_{i j}$ и $e_{j} b e_{i} \in T_{j i}$. Поэтому $\left(e_{j} b e_{i}\right)\left(e_{i} a e_{j}\right) \in T_{j j}$. Кроме того, $\left(e_{i} a e_{j}\right)\left[\left(e_{j} b e_{i}\right)\left(e_{i} a e_{j}\right)\right]=\left(e_{i} t e_{i}\right)\left(e_{i} a e_{j}\right)$, значит можно положить $e_{j} u(i, j) e_{j} \equiv\left(e_{j} b e_{i}\right)\left(e_{i} a e_{j}\right)$.

Так как $e_{j} A$ - цепной $A$-модуль, то конечное множество $\left\{e_{j} u(i, j) e_{j} A\right\}_{i=1}^{n}$ имеет минимальньй элемент, которьй обозначим через $u_{j}$. Тогда $u_{j}=e_{j} u_{j} e_{j}$ и $\left(e_{i} a e_{j}\right) u(j) \in$ $\left(e_{i} t e_{i}\right) A$ при $i=1, \ldots, n$. Пусть $u=u_{1}+\cdots+u_{n} \in T$. Тогда $a u \in t A$. Поэтому $T-$ правое множество Оре.

Аналогично, $T$ является левьм множеством Оре. Поэтому кольцо частных $Q$ кольца $A$ относительно $T$ существует. По лемме $2.2(3) \quad Q$ - полуцепное кольцо. По лемме $2.2(2)$ все $e_{i} Q$ - цепные $Q$-модули, откуда $e_{i}$ - локальные идемпотенты в $Q$. По лемме $1.7(2)$ все кольца $e_{i} A e_{i}$ и $e_{i} Q e_{i}$ цепные. Оставшиеся утверждения проверяются непосредственно.

(2) Пусть $R$ - множество всех регулярных элементов кольца $A, r \in R$ и $a \in A$. По лемме 2.5 (3) существуют натуральное число $m$ и обратимые элементы $u$ и $v$ кольца $A$ такие, что $(u r v)^{m}=e_{1}(u r v) e_{1}+\cdots+e_{m}(u r v) e_{m}$. Тогда $(u r v)^{m} \in T$ и $e_{i}(u r v) e_{i} \in$ $T_{i}$ для любого $i$. Согласно (1) $T$ - правое множество Оре. Для элементов $u a \in A$ и $(u r v)^{m} \in T$ существуют элементы $b \in A$ и $x \in T$ такие, что $u a x=(u r v)^{m} b$, откуда $a x=$ $r\left(v(u r v)^{m-1} b\right)$. Поэтому $R$-правое множество Оре. Аналогично, $R$ - левое множество Ope. Следовательно, классическое кольцо частных $Q_{\mathrm{cl}}(A)$ кольца $A$ существует. По лемме $2.2(3) Q_{\mathrm{cl}}(A)$ - полуцепное кольцо.

Полуцепное кольцо частных $Q$ содержится в $Q_{\mathrm{cl}}(A)$. Чтобы доказать равенство $Q=$ $Q_{\mathrm{cl}}(A)$, достаточно доказать, что любой регулярный элемент $q$ кольца $Q$ обратим в $Q$. Так как $Q$ - полуцепное кольцо, по лемме $2.5(3)$ существуют натуральное число $k$ и обратимые элементы $x, y$ кольца $Q$ такие, что $(x q y)^{k}=e_{1}(x q y) e_{1}+\cdots+e_{k}(x q y) e_{k}$. Все $e_{i}(x q y) e_{i}$ - регулярные элементы в $e_{i} Q e_{i}$. Согласно (1) $e_{i} Q e_{i}-$ классическое кольцо частных кольца $e_{i} A e_{i}$, откуда элементы $e_{i}(x q y) e_{i}$ обратимы в $e_{i} Q e_{i}$. Поэтому $(x q y)^{k}$ обратимо в $Q$. Кроме того, $x$ и $y$ обратимы в $Q$. Тогда $q$ обратимо в $Q$. 
(3) Согласно (1) все $e_{i} A e_{i}$ - цепные кольца и $e_{i} Q e_{i}-$ классическое кольцо частных кольца $e_{i} A e_{i}$ для любого $i$. По лемме 2.6(2) все кольца $e_{i} Q e_{i}$ являются артиновыми. По лемме $1.7(2)$ все $e_{j} Q e_{i}$ - цепные правые $e_{i} Q e_{i}$-модули и цепные левые $e_{j} Q e_{j}$-модули. По лемме 2.4(3) $Q$ - артиново кольцо. Оставшиеся утверждения следуют из (2).

Теорема 2 следует из леммы 2.7 (3).

\section{3. Доказательство теоремы 3.}

ЛЕмма 3.1. Пусть $Q$ - конечнопорожсденый проективный правый модуль над полуцепным кольцом $A$.

(1) Пусть $R$ - любой ненулевой конечнопорожденный подмодуль в $Q u h: Q \rightarrow$ $Q / R$ - естественный эпиморфизм. Тогда существуют прямое разложение $Q=$ $Q_{1} \oplus \cdots \oplus Q_{n}$ и натуральное число $m \leqslant n$ такие, что $R=R \cap Q_{1} \oplus \cdots \oplus R \cap Q_{m}-$ существенный подмодуль в $Q_{1} \oplus \cdots \oplus Q_{m}, R \cap Q_{i} \neq 0(1 \leqslant i \leqslant m), h\left(\bigoplus_{i=1}^{m} Q_{i}\right) \cong$ $\bigoplus_{i=1}^{m} Q_{i} /\left(R \cap Q_{i}\right)$ - сингулярный модуль и $Q / R=P \oplus h\left(\bigoplus_{i=1}^{m} Q_{i}\right)$, дде $P$ - получепной проективный конечнопорохденный модуль такой, что $Q=P \oplus\left(\bigoplus_{i=1}^{m} Q_{i}\right)$, $u h(P) \cong P$ - полуцепной проективный конечнопорожсденный подмодуль в $Q / R$. Кроме того, либо $m=n$ и $P=0$, либо $m<n u P=Q_{m+1} \oplus \cdots \oplus Q_{n}$.

(2) Если $S$ - подмодуль в $Q$, mо $Q / S=T+U$, где $T$ - сингулярный конечнопорожденный модуль, $U$ - получепной проективный конечнопорожденный модуль.

ДокАЗАтЕльство. (1) По теореме 25.3.3 из [7] существуют прямое разложение $Q=$ $Q_{1} \oplus \cdots \oplus Q_{n}$ и натуральное число $m \leqslant n$ такие, что $R=R \cap Q_{1} \oplus \cdots \oplus R \cap Q_{m}$, $R_{i} \equiv R \cap Q_{i} \neq 0$ при $i=1, \ldots, m$. Так как ненулевые подмодули $R_{i}$ цепных модулей $Q_{i}$ являются существенньми в $Q_{i}$, то $R=R \cap Q_{1} \oplus \cdots \oplus R \cap Q_{m}$ - существенный подмодуль в $Q_{1} \oplus \cdots \oplus Q_{m}$. Поэтому $h\left(\bigoplus_{i=1}^{m} Q_{i}\right) \cong \bigoplus_{i=1}^{m} Q_{i} / R_{i}-$ сингулярньй модуль. Если $m=n$, то оставшиеся утверждения очевидны. Пусть $m<n$ и $P \equiv Q_{m+1} \oplus \cdots \oplus Q_{n}$. Тогда $P$ - конечнопорожденньй проективный модуль, $Q=P \oplus\left(\bigoplus_{i=1}^{m} Q_{i}\right)$ и $Q / R=$ $P \oplus h\left(\bigoplus_{i=1}^{m} Q_{i}\right)$. Так как $P \cap \operatorname{Ker}(h) \subseteq P \cap\left(Q_{1} \oplus \cdots \oplus Q_{m}\right)=0$, то $h(P) \cong P-$ конечнопорожденньй проективный подмодуль в $Q / R$. Модуль $P$ изоморфен прямой сумме локальных модулей над цепным кольцом $A$. Поэтому $P$ полуцепной.

(2) Так как $Q$ - конечномерньй модуль, то $S$ - существенное расширение конечнопорожденного модуля $R$. Пусть $h: Q \rightarrow Q / R$ - естественньй эпиморфизм. Тогда $h(S)-$ сингулярньй модуль. Без ограничения общности можно считать, что модуль $Q / R$ не является сингулярным. Согласно (1) существуют прямое разложение $Q=Q_{1} \oplus \cdots \oplus Q_{n}$ и натуральное число $m<n$ такие, что $R$ - существенньй подмодуль в $Q_{1} \oplus \cdots \oplus Q_{m}$, $h\left(Q_{1} \oplus \cdots \oplus Q_{m}\right) \equiv T_{1}-$ сингулярньй конечнопорожденный модуль и $Q / R=P \oplus T_{1}$, где $P \equiv Q_{m+1} \oplus \cdots \oplus Q_{n}-$ полуцепной проективньй конечнопорожденньй модуль такой, что $P \cong h(P) \subseteq Q / R$. Пусть $w: Q \rightarrow Q / S$ - естественньй эпиморфизм. Так как $P \cap R=0$ и $S$ - сушественное расширение для $R$, то $P \cap S=0$. Поэтому $P \cong w(P) \subseteq Q / S$. Пусть $T \equiv w\left(Q_{1} \oplus \cdots \oplus Q_{m}\right), U \equiv w(P)$. Тогда $Q / S=T+U$, где $U$ - полуцепной проективньй конечнопорожденньй модуль. Так как $T$ - гомоморфньй образ сингулярного конечнопорожденного модуля $h(S)+T_{1}$, то $T$ - сингулярньй конечнопорожденньй модуль.

ПРЕДЛОЖЕНИЕ 3.2. Каждый конечнопорожденный модуль над полуцепным кольиом является суммой (необязательно прямой) сингулярного конечнопорохденного модуля и полуцепного проективного конечнопорохденного модуля. 
ДоКАЗАТЕЛЬСТВО. Предложение следует из леммы 3.1(2) и того, что каждьй конечнопорожденньй модуль изоморфен фактормодулю конечнопорожденного свободного модуля.

СлЕДСТВИЕ 3.3. Пусть $A$ - полуцепное кольцо. Тогда

(1) каждый конечнопорожденный несингулярный $A$-модуль является полуцепным проективным модулем [5];

(2) каждый неразложимый конечнопорожденный несингулярный $A$-модуль является иепным проективным ииклическим модулем;

(3) каждый равномерный несингулярный $A$-модуль $M$ является иепным модулем и, следовательно, все неразложимые инбективные несингулярные A-модули являются иепными модулями [13].

ДокАЗАТЕЛЬСТво. (1) следует из предложения $3.2 ;(2)$ следует из (1); (3) следует из (2).

Лемма 3.4. Пусть $A-$ кольцо и $M-$ правый $A$-модуль, не являющийся сингулярным модулем. Тогда $M$ содержит изоморфную копию некоторого ненулевого правого идеала кольца $A$.

Кроме того, если $A$ - конечномерное справа кольцо, то $M$ содержит изоморфную копию ненулевого равномерного правого идеала кольца $A$.

ДокАЗАТЕЛЬСТво. По предположению существует ненулевой элемент $m \in M$ такой, что правый идеал $r(m)$ кольца $A$ не является существенньм. Пусть $h: A_{A} \rightarrow$ $m A$ - естественный эпиморфизм и $B$ - ненулевой правый идеал кольца $A$ такой, что $B \cap r(m)=0$. Так как $B \cap \operatorname{Ker}(h)=0$, то модуль $B_{A}$ изоморфен ненулевому подмодулю $h(B)$ модуля $M$. Если $A$ конечномерно справа, то $B$ содержит ненулевой равномерньй правьй идеал $C$, значит $M$ содержит $h(C)$.

Лемма 3.5. Пусть $A$ - конечномерное справа кольцо. Тогда

(1) все прямые суммы несингулярных инбективных правых $A$-модулей инвективны;

(2) каждый несингулярный инбективный правый $A$-модуль $M$ является прямой суммой равномерных инбективных модулей.

ДокаЗАТЕЛьство. (1) Пусть $M_{A}=\bigoplus_{i \in I} M_{i}$, где все $M_{i}$ - несингулярные инъективные модули и $f: B_{A} \rightarrow M$ - гомоморфизм из правого идеала $B$ кольца $A$. Так как $A$ конечномерно справа, то $B_{A}$ - существенное расширение конечнопорожденного правого идеала $C$ кольца $A$. Существует конечное подмножество $F$ множества индексов $I$ такое, что конечнопорожденньй подмодуль $f(C)$ модуля $M$ содержится в $\bigoplus_{i \in F} M_{i} \equiv N$. Так как $N$ - конечная прямая сумма инъективных модулей, то $N$ инъективен. Поэтому ограничение $f$ на $C$ продолжается до гомоморфизма $g: A_{A} \rightarrow N \subseteq M$. Так как $(f-g)(C)=0$ и $B_{A}$ - существенное расширение модуля $C$, то $(f-g)(B)-$ сингулярный модуль. Кроме того, $(f-g)(B)$ - подмодуль несингулярного модуля $M$. Поэтому $(f-g)(B)=0$. Тогда $g-$ продолжение $f$, откуда модуль $M$ инъективен.

(2) По лемме 3.4 каждьй ненулевой подмодуль модуля $M$ содержит ненулевой равномерный подмодуль. Поэтому любое ненулевое прямое слагаемое инъективного модуля $M$ имеет ненулевое равномерное инъективное прямое слагаемое. По лемме Цорна $M$ является сушественным расширением прямой суммы $N$ равномерных инъективных 
модулей $N_{i}(i \in I)$. Согласно (1) модуль $N$ инъективен. Так как $N$ - существенное прямое слагаемое модуля $M$, то $M=N$.

ДокАЗАТЕЛЬСтво тЕОРЕмы 3. Первое утверждение следует из предложения 3.2. Тот факт, что каждый несингулярный инъективный правый модуль над полуцепным кольцом является полуцепным модулем, следует из леммы $3.5(2)$ и следствия $3.3(3)$. Пусть $N$ - произвольный несингулярньй $A$-модуль. По следствию $3.3(1)$ все конечнопорожденные подмодули модуля $N$ являются проективными и, в частности, плоскими модулями. Тогда $N$ - плоский модуль (см. [14, 39.12]).

\section{СПИСОК ЦИТИРОВАННОЙ ЛИТЕРАТУРЫ}

[1] Singh S. Serial right Noetherian rings // Canad. J. Math. 1984. V. 36. № 1. P. 22-37.

[2] Туганбаев А. А.Дистрибутивные кольца и модули // Матем. заметки. 1990. Т. 47. № 2. C. 115-123.

[3] Warfield R. B. Bezout rings and serial rings // Comm. Algebra. 1979. V. 7. P. 533-545.

[4] Dubrovin N. I. The rational closure of group rings of left-ordered groups. Gerhard Mercator Universität Duisburg Gesamthochschule, 1994.

[5] Warfield R. B. Serial rings and finitely presented modules // J. Algebra. 1975. V. 37. №3. P. 187-222.

[6] Goodearl K.R. Ring Theory. Nonsingular Rings and Modules. New York-Basel: Marcel Dekker, 1976.

[7] Фейс К. Алгебра: кольца, модули и категории. Т. 2. М.: Мир, 1979.

[8] Small L. W. Semihereditary rings // Bull. Amer. Math. Soc. 1967. V. 73. № 5. P. 656-658.

[9] Chatters A. W. The restricted minimum condition in Noetherian hereditary rings // J. London Math. Soc. 1971. V. 4. P. 83-87.

[10] Туганбаев А. А. Дистрибутивные справа или слева кольца // Матем. заметки. 1995. T. 58. № 4. C. 604-627.

[11] Туганбаев А. А. Кольца, над которыми каждый модуль является прямой суммой дистрибутивных модулей // Вестн. МГУ. Сер. 1. Матем., мех. 1980. №1. С. 61-64.

[12] Дрозд Ю.А. Об обобщенно однорядных кольцах // Матем. заметки. 1975. Т. 18. №5. C. $707-710$.

[13] Upham M. H. Serial rings with right Krull dimension one // J. Algebra. 1987. V. 109. P. 319-333.

[14] Wisbauer R. Foundations of Module and Ring Theory. Philadelphia: Gordon and Breach, 1991. 